\title{
Implementing bezier surface interpolation and N.N in shape reconstruction and depth estimation of a $2 \mathrm{D}$ image
}

\author{
Mohamed Ibrahim Shujaa, Ammar Alauldeen Abdulmajeed \\ Electrical Enginerring Technical College, Department of Computer Engineering, Middle Technical University, Iraq
}

\begin{tabular}{l} 
Article Info \\
\hline Article history: \\
Received Feb 19, 2019 \\
Revised Apr 22, 2019 \\
Accepted Jun 5, 2019 \\
\hline
\end{tabular}

\section{Keywords:}

Bezier spline surface Mapping 2D curved object into $3 \mathrm{D}$ curved object Object depth estimation

\begin{abstract}
This paper considers a 2D image depth estimation of an object and reconstructed it into a $3 \mathrm{D}$ object image. The $2 \mathrm{D}$ image is defined by slices contains asset of points that are located along the object contours and within the object body. The depth of these slices are estimated using the neural network technique (N.N), where five factors (slice length, angle of incident light and illumination of some of point that located along the 2D object, namely control points)are used as inputs to the network the estimated depth of the slice are mapped into a 3D surface using the interpolation technique of the Bezier spleen surface. The experimental results showed an effective performance of the proposed approach.
\end{abstract}

Copyright $@ 2019$ Institute of Advanced Engineering and Science. All rights reserved.

\section{Corresponding Author:}

Mohamed Ibrahim Shujaa,

Department of Technical Computer Engineering,

Electrical Enginerring Technical College, Middle Technical University (MTU), Iraq.

Email: drshujaa71@gmail.com

\section{INTRODUCTION}

Object depth estimation from 2D image is one of the most active research topic because it's the basic problem in computer vision and has important application in robotics, pattern recognition, graphic and machine vision.3D image construction is the challenging problem and many researchers have been performed to resolve it. In [1] the author have proposed a supervised learning approach for depth estimation using a 3D scanner to collect the training data that used to model a conditional distribution of depth that was gave a monocular image features. Several methods are used for detection object presented in [2].

Other investigator, [3] have developed a simultaneous phase-shifting technique using an innovating colour fringe pattern with multiple triangular modulation for 3D vision system. Shape and depth from shading techniques for 3D surface reconstruction were presented in $[4,5]$. However shading technique is only valid to acquire object height information for the direction associated with the incident light and the generated object shadow.

Many works on 3D construction have been focused on 2D images. In [6] the object was projected through a grid of pseudorandom encoded structured light and determines a set of references pixels in two simultaneous view of the same object using two cameras. While in [7] the model of 3D object that obtained from 2D image are based on human-computer interaction. The human was provided with much visual assistance as possible to make a correct input and verify it.

Constructing a 3D graphical image model was proposed in [8], where six surfaces including front, back, left, right, top, and bottom view image were used to form the model and the colour matrixes. Reconstruction methods from contours line was provided in [9]. In the model, zhong and coauthors suggested to rid some of redundant point on every contour and interpolate them by using cubic Bezier line curve. In [10] the author suggested to represent a geometric 3D shape as a probability distribution of binary variable on a 3D voxel grid using (Convolution Deep Belief Network). Generating a 3D image from a consecutive of 
$2 \mathrm{D}$ image has been investigated by [11]. 3D face reconstruction has been introduced in [12]. Where the author integrated variant face pose in order to reconstruct a 3D image face model.

The suggested model in this paper is different from others by proposing a new methodology to estimate the depth of an object. The model takes advantage of light distribution over an object, where their distribution alters based on object depth as well as angle of the incident light. In our model, asset of points in assigned in the 2D image that lays inside the object and along the contour. We referred to this point as control points. Neural network technique is used to estimate the depth, where object width illumination and angle $(\theta)$ of the incident light are considered. The $2 \mathrm{~d}$ object image is reconstructed into $3 \mathrm{D}$ object using Bezier spline surface. The proposed approach was tested on some 2D image and showed tolerable results.

\section{CAMERA-OBJECT SETUP}

In order to get 3D complex object with deferent depth AUTO CAD software has been utilized to form various shape with variant depth. The $2 \mathrm{D}$ image are captured by linking camera with a target point light in which both are located perpendicular to the object at $(150) \mathrm{cm}$. Figure 1 represent a sketch of the cameraobject setup structure whereas, Land $\mathrm{R}_{\mathrm{d}}$ are the camera height and the depth of the object respectively.

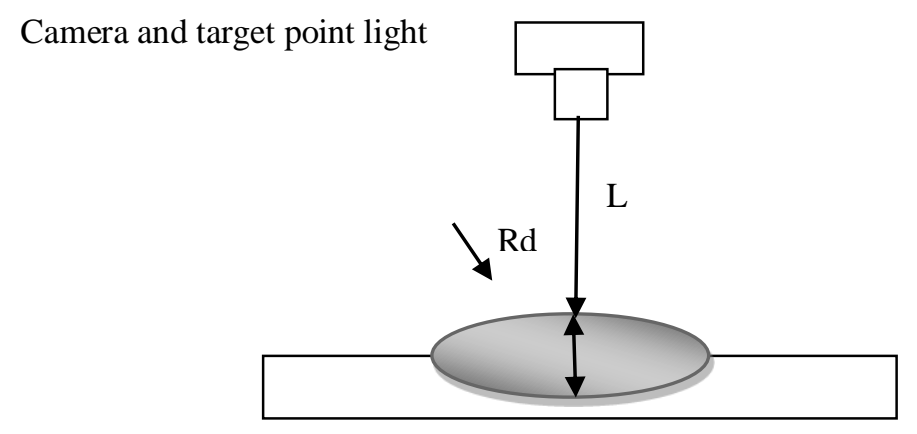

Figure 1. The implementing structure

The resolution of the captured image is 640-480. A set of image pre-processing techniques are applied including: digital image de noising, thresholiding and edge detection that presented in [13], and the contour tracing to extract the edge as a set of connected pixel as presented in [14]. We can also use K-Mean classification (KM) to classify defied $\mathrm{K}$ group for data [15]. In this paper cylinder object with radius (15) $\mathrm{cm}$ and height of $(40) \mathrm{cm}$ is used as experimental sample to illustrate how to construct the 3D object image through applying the proposed stage of the methodology, where figure (2-a) and (2-b) represent the image of the used cylinder and its contour respectively.

\section{RESEARCH METHODS}

The proposed method is divided into three main parts, setting the control point, neural network technique, and construction of 3-D object via Bezier surface interpolation. The parts are described in the Figure 2.

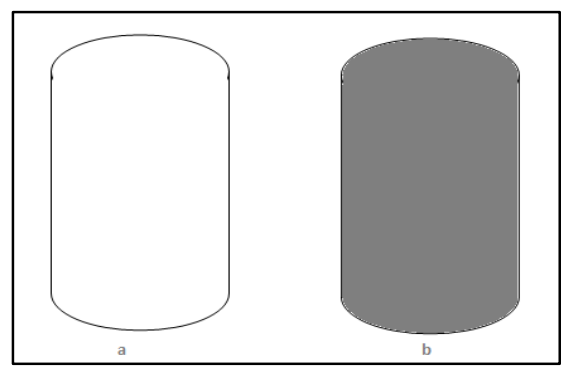

Figure 2. Cylinder object. (a) The 2D image (b) The contour of image 


\subsection{Set the control point}

The control point setting is a substantial issue of determining the object depth, it consist of two steps. In the first step, the object contour is divided into halves, right and left. Figure 3 shows the halves of the cylinder's contour, where each one includes two types of points, the edge and the halves control points. The edge control points are located along the object contour while the halve control points are placed in the middle of the halves. The spaces between the edge control points are equal to the space of the halves control points. Experimentally (10) pixels are convenient distance between control point where it gives an accurate trace for object shape.

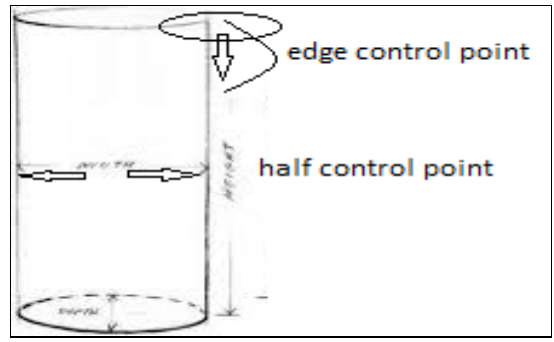

Figure 3. Two halves contour of the cylinder

The right and the left side of the object contour can be represented by two matrixes Rs and Ls respectively, where each element contains the value of $(x, y)$ coordinate for the control point. The right halves side:

$$
\mathrm{Rs}=\left[\mathrm{r}_{\mathrm{sij}}\right] \quad \mathrm{i}: \rightarrow \mathrm{n}, \mathrm{j}: 1 \rightarrow \mathrm{m} .
$$

The left halves side :

$$
\mathrm{Ls}=\left[1_{\mathrm{sij}}\right] \quad \mathrm{i}: \rightarrow \mathrm{n}, \mathrm{j}: 1 \rightarrow \mathrm{m} .
$$

where $\mathrm{n}$ is the number of control points while $\mathrm{m}$ is the type number of these points and equals 2 , edge and halves. In the next steps of the setting methods, Rs an Ls are combined in one matrix Ts as shown in Equation (3).

$$
\mathrm{Ts}=[\mathrm{Rs} \mathrm{Ls}]_{\mathrm{nxk}}
$$

$\mathrm{K}$ is the number of whole types of the control points and equals 4 .

In our method, a slice will be referred to a set of points that have the same level where each slice have 4 control points. These points are connected one to another to construct the slice shape, where Figure 4 shows the slice shape of the cylinder that has (33) slices of length of (288) pixels. The cylinder ends are demonstrated with (12) slice while the cylinder body represent with (21) slice. Obviously the slice that constituent the cylinders body are equal width while the slice in the other side are very close compare with the slice of the body, in spite of all distance between the cylinder slices are equal to 10 pixel as mentioned previously. This is due to the convergence between contours sides (left and right).

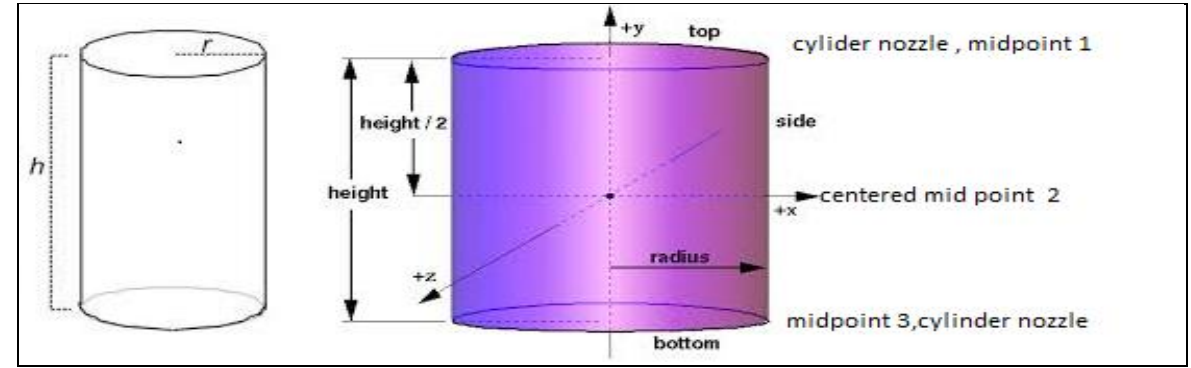

Figure 4. (a) Cylinder shape; (b) Shape of the object as slices and set of control points 


\subsection{Neural network technique}

The neural network represents a powerful data processing technique that has reached maturity and broad application $[16,17]$. In this paper the neural network technique is used to estimate the value of depth. Here a network with five inputs, two hidden layer and one output is proposed. The hidden layer is composed from eight neurons. The height of the slice, the illumination of the slice midpoint, to half control point illumination and the incident light angle $(\theta)$ represent the five input of the network while the depth represent the output. The first input portrays the number of the pixels in the slice while the intensity value of the midpoint and the two half control are used as second, third and fourth input respectively. The fifth input $(\theta)$, that is shown in Figure 5 can be defined as the angle between the perpendicular incident light on the centered midpoint and the incident light on the required midpoint as in Equation (4).

$$
\theta_{\mathrm{k}}=\tan ^{-1}\left(\frac{(Y k \mid Y) n / 2}{L}\right) \mathrm{k}: 1 \rightarrow \mathrm{n} .
$$

where $\left(\mathrm{Y}_{\mathrm{k}}-\mathrm{Y}_{\mathrm{n} / 2}\right)$ is vertical distance between the slice midpoint and the cantered mid point and $\mathrm{L}$ is the perpendicular distance of the incident light on the entered midpoint.

The proposed network is verified using the matlab program, where back propagation learning algorithm is used with 0.05 learning rate. All neurons are set to use a sigmoid activation function. The network is trained using 300 sets that selected to cover many depth cases for different curved shape.

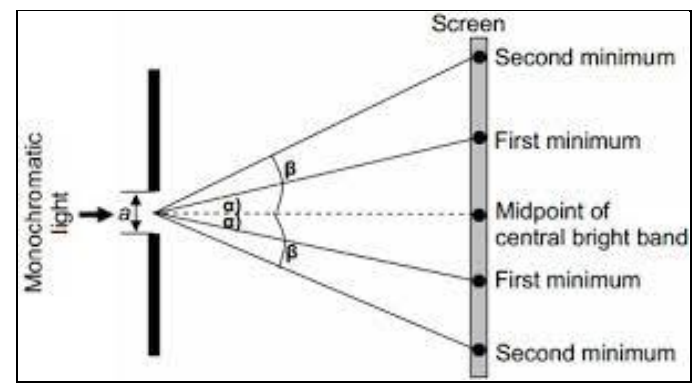

Figure 5. The angle of midpoint incident light

\subsection{Construct the 3D shape}

Bezier surface are a species of a mathematical spline used in computer graphics, computer-aided design and finite element modeling. It is defined by a rectangular grid of control point; it is anchored at the four corner point and employs the other grid points to determine its shape as pointed in [18-20].

In this work the Bezier surface interpolation is utilized to construct the $3 \mathrm{D}$ shape where the value of the estimated depth of each slice will be used as the $\mathrm{z}$ value for the half control point of the same slice while the depth of the edge control point will assign to zero Equation (3) is divided into three (4*n) sub-matrices, $[21,22]$, where each one has a simple component of coordinates. These matrices are $C_{x}, C_{y}$, and $C_{z}$ which referred to $\mathrm{x}, \mathrm{y}$, and $\mathrm{z}$ component respectively. Equation (5) shows the matrix form of $\mathrm{x}$ component where $\mathrm{y}$ and $\mathrm{z}$ component can be represented in the same way.

$$
\left[\begin{array}{cccc}
\sec 1 & \text { sec } 2 & \text { sec } 3 & \text { sec } 4 \\
x(1,1) & x(1,2) & x(1,3) \ldots \ldots x(1, n) \\
x(2,1) & x(2,2) & x(2,3) \ldots \ldots x(2, n) \\
x(3,1) & x(3,2) & x(3,3) \ldots \ldots x(3, n) \\
x(4,1) & x(4,2) & x(4,3) \ldots \ldots x(4, n) \\
x(5,1) & x(5,2) & x(5,3) \ldots \ldots x(5, n)
\end{array}\right]
$$

The 3D shape is constructed using a bi cubic Bezier matrix surface where the bi cubic Bezier matrix is given by a grid of ( $4 * 4)$ control point. Obviously, each four slices represent 16 control point or one Bezier patch so for an object shape that has more than four slices, a single surface patch does not enough to cover all objects details. Hence the object surface will be described through several patch joint together through continuity patch to ensure that smoothness of the surface. The main matrix $\mathrm{C}_{\mathrm{x}}, \mathrm{C}_{\mathrm{y}}$ and $\mathrm{C}_{\mathrm{z}}$ are divided into sub matrix where each of them has four slices (16 control point). The number of sub matrix is depending on the 
number of slices as shown in $[23,24]$. The bi cubic Bezier surface can be shown in matrix form through equation (6).

$$
\mathrm{S}(\mathrm{u}, \mathrm{v})=[\mathrm{U}][\mathrm{N}][\mathrm{CP}][\mathrm{N}][\mathrm{V}]
$$

Hence the $4 * 4$ matrix [CP] stores the control points, [N] contains the Bernstein polynomial coefficients $[\mathrm{U}]=\left[\begin{array}{lll}\mathrm{u}_{3} & \mathrm{u}_{2} & \mathrm{u}_{1}\end{array}\right]$ and $\left[\mathrm{v}_{3} \mathrm{v}_{2} \mathrm{v}_{1}\right]$ as mentioned in [25]. The construction of 3-D matrix shape through a set of Bezier patches and continuity patch is Clearfield in Figure 6, where the cylinder shape with 33 slices are portrayed as a wireframe object that consist from six Bezier patch and five continuity patch.

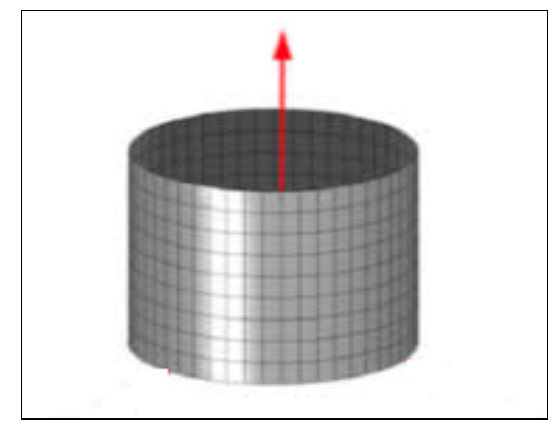

Figure 6. Bezier surface of the cylinder

\section{RESULTS AND ANALYSIS}

In order to evaluate the performance of our methodology we selected four 2-D images of different shape including cone, bowel, vase, and sphere. These images contain Varity of curves and depth which makes the construction of 3D image more complex and challenged. Figure 7 represent the tested objects where the picture of the left column is the original 2-D object image while those of right column are the constructed 3D object shape image. The cone and bowl image are represented in the Figure 7(a-L) and (b-L) respectively, each of them has different depth value and a total length of $40 \mathrm{~cm}$. In the first object, the depth of upper ends starts from $(25) \mathrm{cm}$ and growth down to $(50) \mathrm{cm}$ at the lower end, while the second object, the depth starts from $(30) \mathrm{cm}$ where it is enlarging to reach $(50) \mathrm{cm}$ and then decrease until $(15) \mathrm{cm}$ at the end of the object. The cone and bowl from (64) slice that translated into a wire frame Bezier surface with (11) Bezier patch connected with (10) continuity patch as shown in the Figure 7(aR) and (bR) respectively.

Figure 7(c-L) shown the image of vase object. It has disparity values of depth between $(20-45) \mathrm{cm}$ constructed a shape with (56) slices .these slices are translated into (10) Bezier patch connected by (10) continuity patches. The whole patches produced a wire-frame Bezier surface of the vase objects with various depths as illustrated in Figure 7(c-R).

Finally the 2-D image of the sphere object with (15) $\mathrm{cm}$ radius is shown in Figure 7(d-L). It is transformed to a shape of (22) slices with (4) Bezier patches connected by (3) continuity patches as represented in Figure 7(d-R). The proposed methodology is evaluated through the experimental results that indicate verity value of error depending on complexity of the tested shape. The mean error value $\mathrm{E}$ is calculated though Equation (7).

$$
\mathrm{E}=\frac{1}{N} \sum_{i=0}^{n} \mathrm{e}
$$

where $\mathrm{N}$ is the number of sample and $\mathrm{e}$ is the error value of the estimated depth that calculated for each sample as illustrated in Equation (8).

$$
\mathrm{e}=\sum_{j=0}^{m} \frac{\mathrm{Rdj}-\mathrm{Edj}}{\mathrm{Rdj}}
$$

where $\mathrm{M}$ is the number of slices in the object shape, $R d, E d$ are the real depth and the estimated depth of the object respectively. For the five tested samples including cylinder, cone, bowl, vase and sphere objects the mean error value doesn't exceed (3\%). 


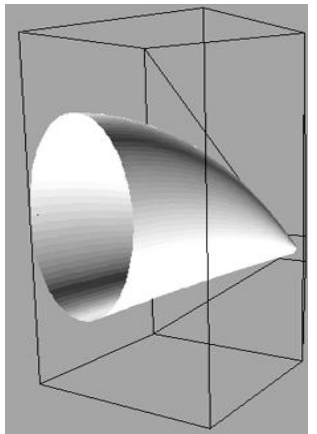

(a-L)

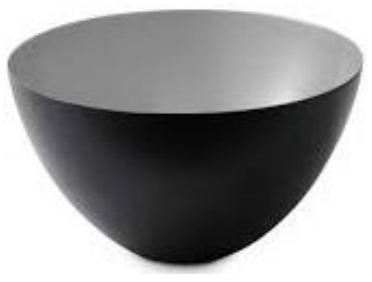

(b-L)

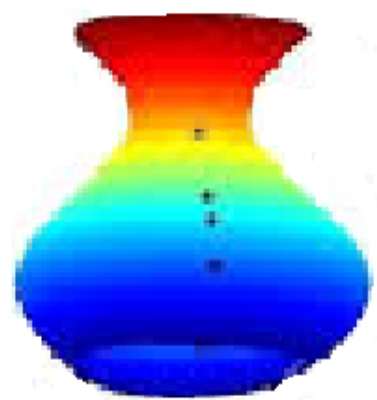

(c-L)

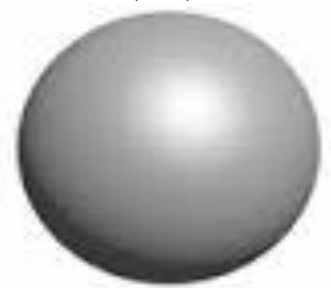

$(\mathrm{d}-\mathrm{L})$

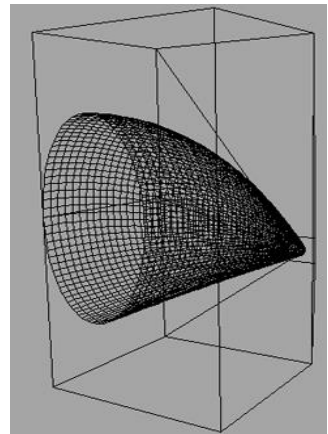

(a-R)

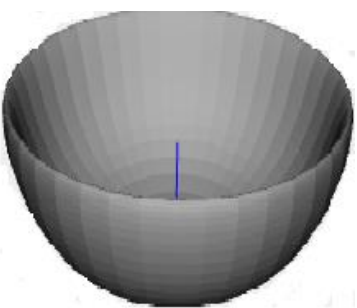

(b-R)

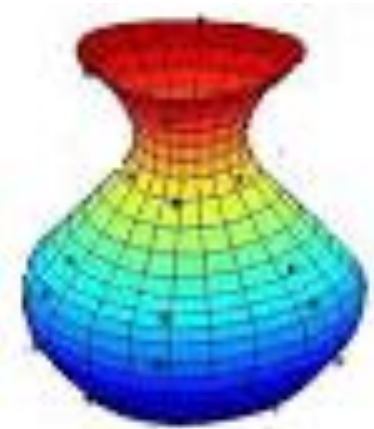

(c-R)

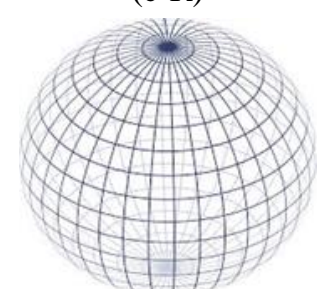

$(\mathrm{d}-\mathrm{R})$

Figure 7. The $2 \mathrm{~d}$ image of cone, bowl, vase and sphere and their 3-d Bezier surface image

\section{CONCLUSTION}

In this paper the depth of an object in a 2D image is estimated and utilized in the 3D shape construction. A still camera with a target point light is used to capture the $2 \mathrm{D}$ image processing technique to get the contour of the object. The object contour is divided in two parts (left and right) sides. Two types of control points named edge control point and half control point are located on the both side. The control points help in determining the slice shape of the object. The neural network technique is used to get an estimation value of for object depth based on a set of inputs value including the length of the slices. Midpoint illumination, two half control points illumination and the incident light angle. The Bezier surface is used to construct the 3D image shape of the 2D image object based on the estimated depth values. In order to evaluate our proposed model, we selected four different objects.( cone, bowl, vase, sphere) where these objects are characterized by varying depth along the object shape. As a consequence, the 3D shape estimation for these objects is a challenge for our proposed model. The result reveals a beneficial estimation in which 
the mean error of whole selected objects is $3 \%$. Our model has a potential to be used in robot-grasping task in which the geometry of $3 \mathrm{D}$ object is reconstructed and then grasping position and orientation could be determined. Another potential is by implementing the model in industrial-CNC machine. This could provide the machine with image based depth estimation to manufacture in $3 \mathrm{D}$ object from a $2 \mathrm{D}$ image.

\section{REFERENCES}

[1] S. Ashutosh, et al., "3D Depth Reconstruction from a Single Still Image," Springer Int J Comput Vis, 2007.

[2] J. Cao, et al., "Object Tracking Based on Multiple Features AdaptiveFusion," Telkomnika Indonesian Journal of Electrical Engineering, vol. 12, pp. 6811-6818, 2014.

[3] C. Liang and N. Xuan, "Real- Time 3D Robot Vision Employment Novel ColorFringe Projection," Proceedings of the $4^{\text {th }}$ international conference on Autonomous Robot and Agent, 2009.

[4] G. Al-Kindi and K. Ali, "Investigation of Object Shadow Utilization in 3D Shape reconstruction using inexpensive Equipment," Transaction in utility and image recognition, vol. 1, 2010.

[5] R. Fabio, et al., "Turning image into 3D model," IEEE single processing magazine, 2008.

[6] L. Phillip, et al., "3D object model recovery from 2D image using structured light," IEEE transaction on instrumentation and measurement, vol. 53, 2004.

[7] M. Yuichi and K. Avinash, "An interactive Frame work for Acquiring vision model of 3D object from 2D image," IEEE Transaction on system Man and cybernetics, vol. 34, 2004.

[8] T. K. Yang, et al., "Rapidly construction a simple 3D graphic model using a consumer user digital still camera," IEEE Transaction on consumer electronics, vol. 52, 2006.

[9] L. Zhong, et al., "Reconstruction from contour line based on bi-cubic Bezier Spline surface," Journal of Zhejiang university-Science A, vol. 7, pp. 1241-1246, 2006.

[10] Z. Wu, et al., "3D shape nets a deep representation of volumetric shape," The IEEE conference of computer vision and pattern recognition (CVPR), pp. 1912-1920, 2015.

[11] D. A. Sponeer, et al., "Methodology for 3D scene reconstruction from 2D image sequences," Us. Patent, 2014.

[12] D. Jianga, et al., "Efficient 3D reconstruction for face recognition," Pattren 2015 recognition, vol. 38, pp. 787-798, 2005.

[13] G. Rafal and W. Richared, "Digital image processing," Third Edition Pearson Education, Inc, 2008.

[14] G. Al-kindi, et al., "An example of two dimensional component inspection using computer vision," Proc. Instn. Mech. Eng., vol. 205, pp.77-83, 1991.

[15] R. Thirumahal and P. A. Deepali, "KNN and ARL Based Imputation to Estimate Missing Values," Indonesian Journal of Electrical Engineering and Informatics, vol. 2, pp. 119-124, 2014.

[16] D. Gerard, "Neural network methodology and application," Springer-verlag Berlin Heidelberg, 2005.

[17] S. David, "Curves and surface for computer graphics," Springer science business media, Inc, 2006.

[18] Yang H., et al., "Image fusion based on multiscale guided filters," IEEE Journal of Optoelectronics Laser, vol. 26, pp. 70-176, 2015.

[19] S. H. Asman and A. F. Abidin, "Comparative Study of Extension Mode Method in Reducing Border Distortion Effect for Transient Voltage Disturbance,” Indones. J. Electr. Eng. Comput. Sci., vol. 6, pp. 628, 2017.

[20] M. H. Jopri, et al., "An Accurate Classification Method of Harmonic Signals in Power Distribution System by Utilising S-Transform,” TELKOMNIKA (Telecommunication Comput. Electron. Control), vol. 15, pp. 62, 2017.

[21] Kumar A. M., "An outcome of periodized small side games with and without mental imagery on playing ability among intercollegiate level soccer players," Indian Journal of Science and Technology, vol. 8, 2015.

[22] Panda S. S. and Jena G., "Image Super Resolution Using Wavelet Transformation Based Genetic Algorithm," Computational Intelligence in Data Mining, vol. 2, pp. 355-361, 2016.

[23] Ganeshbabu T. R., "Computer aided diagnosis of glaucoma detection using digital fundus image," International journal of advances in signal and image sciences, vol. 1, pp. 1-11, 2015.

[24] R. Sugi, "Estimation of Power Quality Indices Using Discrete Wavelet Transform," IEEE, 2016.

[25] Gajdhane A. V. and Deshpande L. M., "Detection of Lung Cancer Stages on CT scans Images by Using Various Image Processing Techniques,” IOSR Journal of Computer Engineering (IOSR-JCE), vol. 16, pp. 28-35, 2014.

\section{BIOGRAPHIES OF AUTHORS}

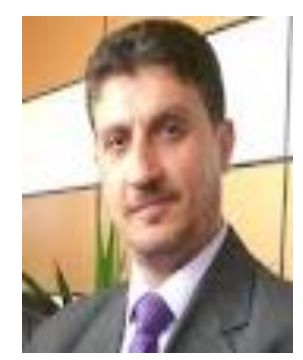

Senior lecturer Dr.Mohamed Ibrahim Shujaa is a member of Electrical Enginerring College, middle Technical University Baghdad (MTU), he has more than 15 years experience in neural network \& network system and in both the industry and academic sectors, and has been involved at various levels of VLSI design such as Encryption and decryption, head motion detection, analogue \& digital circuit design. Currently, he is actively researching new techniques on IOT using DNA and stream cipher to increase the level of encryption. His research interests include raspberry pi and micro controller, low power algorithms and architectures design, and image and video processing, and network. 


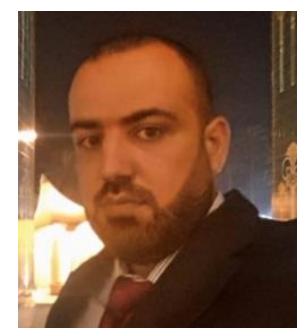

Assistace lecturer Ammar Alauldeen Abdulmajeed is a member of Electrical Enginerring College, middle Technical University Baghdad (MTU),his experience focus on telecommunication network \& fiber optic system and in both the industry and academic sectors, his master degree Msc from university of Malaya 2009 in crystal fiber. Currently, he is working in a reaserch on WSN and laser exploiting photonic. His interest in microwave links design and fiber optic. 